\title{
An ANK1 IVS3-2A>C mutation causes exon 4 skipping in two patients from a Chinese family with hereditary spherocytosis
}

\author{
Xiong Wang ${ }^{1}$, Liyan Mao ${ }^{1}$, Na Shen ${ }^{1}$, Jing Peng ${ }^{1}$, Yaowu Zhu ${ }^{1}$, Qun Hu${ }^{2}$ and Yanjun \\ $\mathbf{L u}^{1}$ \\ ${ }^{1}$ Department of Laboratory Medicine, Tongji Hospital, Tongji Medical College, Huazhong University of Science and Technology, \\ Wuhan, China \\ ${ }^{2}$ Department of Pediatrics, Tongji Hospital, Tongji Medical College, Huazhong University of Science and Technology, Wuhan, \\ China \\ Correspondence to: Qun Hu, email: qunhu2013@163.com
}

Yanjun Lu, email: junyanlu_2000@163.com

Keywords: hereditary spherocytosis; anemia; ANK 1; splenectomy

Received: August 28, $2017 \quad$ Accepted: November 16, $2017 \quad$ Published: December 05, 2017

Copyright: Wang et al. This is an open-access article distributed under the terms of the Creative Commons Attribution License 3.0 (CC BY 3.0), which permits unrestricted use, distribution, and reproduction in any medium, provided the original author and source are credited.

\section{ABSTRACT}

Hereditary spherocytosis (HS) is a congenital hemolytic anemia that affects the cell membrane of red blood cells and is characterized by the presence of sphericalshaped erythrocytes in the peripheral blood film. The clinical manifestation of HS ranges from asymptomatic to severe cases that require transfusion during early childhood. HS is caused by mutations in red blood cell membrane protein encoding genes, including ANK1, EPB42, SLC4A1, SPTA1, and SPTB. Mutations of the ANK1 gene account for $\mathbf{7 5 \%}$ of all HS cases, and these particular mutations are typically inherited in an autosomal dominant manner. In this study, heterozygous an ANK1 IVS32A $>C$ mutation was identified in a 7-year-old girl with Coombs-negative and severe hemolytic jaundice using targeted next-generation sequencing (NGS) and Sanger sequencing. Spherocytes were observed in a peripheral smear. Osmotic fragility was increased, and glucose-6-phosphate dehydrogenase (G6PD) activity was normal. A genetic mutation screen for $\alpha-$ and $\beta$-thalassemia was negative. Autoimmune antibody tests were negative. Both the girl and her affected father received a splenectomy. Patient-derived peripheral blood mononuclear cells showed skipping of exon 4 in the mRNA, which confirmed the splicing mutation effect of the ANK1 IVS3-2A>C mutation. Moreover, the anemia was ameliorated after splenectomy. Our results demonstrate that the ANK1 IVS3-2A>C mutation may lead to exon 4 skipping of the ANK1 gene and cause HS.

\section{INTRODUCTION}

Hereditary spherocytosis (HS) is a common form of inherited hemolytic anemia that has a prevalence ranging from 1:2,000 to 1:5,000 in Caucasians and 1:100,000 in Chinese individuals $[1,2]$. It is caused by defects in red blood cell membrane proteins, such as ankyrin, protein 4.2 , band 3 protein, $\alpha$-spectrin, and $\beta$-spectrin, which are encoded by the ANK1, EPB42, SLC4A1, SPTA1, and $S P T B$ genes, respectively [3]. The main clinical manifestations of HS are highly variable, including signs of anemia, jaundice, gallstones, and splenomegaly [4].
Approximate $75 \%$ of all HS cases are inherited in an autosomal dominant manner. Management of HS patients may include blood transfusions and splenectomy to reduce hemolysis and clinical symptoms [3].

The ANK1 gene is located on chromosome 8p11.1 and encodes several alternatively spliced isoforms [5]. Mutations in the ANK1 gene are responsible for the majority of all HS cases, followed by mutations in the SLC4A1 and SPTB genes. Ankyrin consists of a multiple ankyrin repeat $\mathrm{N}$-terminal domain, a spectrin-binding center region, and a regulatory $\mathrm{C}$-terminal domain. The major function of ankyrin is to stabilize the membrane 
structure by interacting with spectrin, protein 4.2, and band 3 protein. Ankyrin provides a high-affinity linkage between the spectrin-actin based membrane skeleton and the red blood cell membrane [6]. Next-generation sequencing (NGS) has been widely used to perform genetic diagnosis of hemolytic anemia, including HS [7].

In this study, a heterozygous $A N K 1$ IVS3-2A $>C$ mutation was identified by NGS and Sanger sequencing in two patients from a Chinese family, both of whom received a splenectomy. Patient-derived peripheral blood mononuclear cells showed skipping of exon 4 in the mRNA.

\section{RESULTS}

\section{Clinical features of a Chinese family with HS}

A 7-year-old girl with anemia, jaundice, and splenomegaly was diagnosed with HS when she was 1 year of age according to her clinical symptoms, laboratory tests, and a positive family history. Osmotic fragility was increased, and G6PD activity was normal. A genetic mutation screen for $\alpha$ - and $\beta$-thalassemia was negative. Autoimmune antibody tests were negative. Sphericalshaped erythrocytes were found in the peripheral blood film. She recently received a splenectomy due to severe anemia, and the anemia was ameliorated after the splenectomy. Her affected father was diagnosed with HS when he was young and received a splenectomy when he was 10 years old. The family tree is shown in Figure 1A, and the laboratory tests are summarized in Table 1.

\section{NGS output and coverage}

All coding exons and adjacent intronic regions of HS-related genes were sequenced on the Ion torrent PGM, and achieved an average output of 822,775 mapped reads and $98.65 \%$ on-target specificity. The mean depth was 2,282 folds. All target bases were covered at least once, $97.11 \%$ were covered at least 100 times, and $93.49 \%$ were covered at least 500 times. The mean uniformity of base

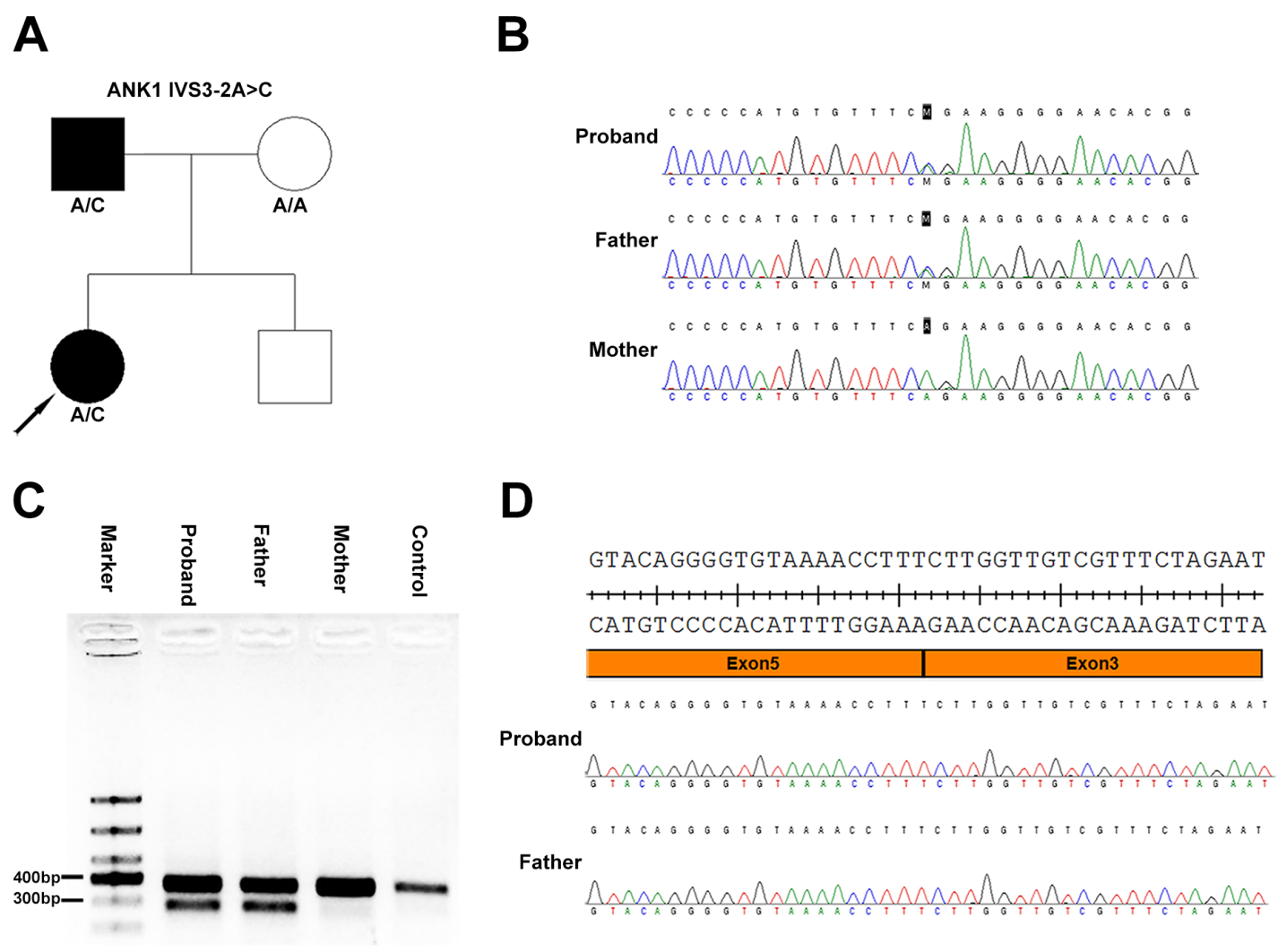

Figure 1: Heterozygous $A N K 1$ IVS3-2A $>C$ mutation in two patients from a Chinese family with hereditary spherocytosis. A. Family tree and the genotype at the ANK1 IVS3-2 position. Squares and circles denote male and female, respectively. Black symbols denote patients with HS. B. Sanger sequencing identified an ANK1 IVS3-2A>C mutation. C. DNA gel of the PCR products spanning exon 2 to 6 from cDNA transcribed from mRNA isolated from patient-derived peripheral blood mononuclear cells. Both the girl's mother and a healthy control yielded a normal $381 \mathrm{bp}$ band, while the girl and her father produced two bands, a normal band and a smaller band. D. Sanger sequencing of the smaller band separated in panel C. These results showed a complete deletion of exon 4 of the $A N K 1$ gene. 
Table 1: Laboratory test results

\begin{tabular}{|l|l|l|}
\hline Test & Result & Reference \\
\hline RBC & $2.58 \times 10^{12} / \mathrm{L}(\downarrow)$ & $4.3-5.8 \times 10^{12} \mathrm{~L}$ \\
\hline MCV & $93 \mathrm{fL}$ & $82-100 \mathrm{fL}$ \\
\hline MCH & $29.5 \mathrm{pg}$ & $27-34 \mathrm{pg}$ \\
\hline MCHC & $31.7 \mathrm{~g} / \mathrm{dL}$ & $31.6-35.4 \mathrm{~g} / \mathrm{dL}$ \\
\hline TBIL & $118.1 \mu \mathrm{mol} / \mathrm{L}(\uparrow)$ & $3.4-20.5 \mu \mathrm{mol} / \mathrm{L}$ \\
\hline IBIL & $112.1 \mu \mathrm{mol} / \mathrm{L}(\uparrow)$ & $\leq 13.3 \mu \mathrm{mol} / \mathrm{L}$ \\
\hline BRD & $6.0 \mu \mathrm{mol} / \mathrm{L}(\uparrow)$ & $0-6.8 \mu \mathrm{mol} / \mathrm{L}$ \\
\hline
\end{tabular}

RBC: red blood cell, MCV: mean corpuscular volume, MCH: mean corpuscular hemoglobin, MCHC: mean corpuscular hemoglobin concentration, TSB: total bilirubin, IBIL: indirect bilirubin, BRD: bilirubin direct.

Table 2: Bioinformatics prediction of splicing mutations by GENIE and MES

\begin{tabular}{|l|l|l|}
\hline Program & Wild type & Mutant \\
\hline GENIE & 4.3 & -6.6 \\
\hline MES & 7.69 & -0.35 \\
\hline
\end{tabular}

coverage is $94.04 \%$.

\section{Mutation detection and Sanger sequencing validation}

NGS identified a total of 56 variants subjected to a process to select pathogenic mutation using the filtration process and annotation. A heterozygous ANK1 IVS3$2 \mathrm{~A}>\mathrm{C}$ mutation was identified.

Sanger sequencing of the ANK1 IVS3-2A $>\mathrm{C}$ mutation was performed on all family members. The results showed that both the patient and her father carried this mutation, while her mother did not (Figure 1B). The genetic analysis for her brother was not available.

Moreover, the $A N K 1$ IVS3-2A $>C$ mutation (hg19: chr8:41585526A $>C$ ) was not found in the $1000 \mathrm{G}, \mathrm{ExAC}$, or HGMD databases.

Amplification of the sequence spanning exon 2-6 of the $A N K 1$ mRNA reversely transcribed cDNA produced only one band in her mother and a healthy control, while the patient and her father produced a normal band and a smaller band separated by DNA agarose gel (Figure 1C). Sequencing of the smaller bands showed skipping of exon 4 in the mRNA (Figure 1D), which led to a direct junction of exon 3 and exon 5 . These results confirm the splicing mutation effect of the ANK1 IVS3-2A $>$ C mutation.

\section{Computational analysis}

The effect of the $A N K 1$ IVS3-2A $>$ C mutation was computationally analyzed using two splice site prediction programs, GENIE and MES, and the result is summarized in Table 2. Both algorithms predicted a weakening of the 3 ' splice-site consensus sequence of exon 4.
Genetic and phenotype association analysis

Genetic tests showed a heterozygous $A N K 1$ IVS3$2 \mathrm{~A}>\mathrm{C}$ mutation in this family, and both patients carried this mutation. Jaundice and splenomegaly were observed in the patients. Consistent with the genetic findings, anemia was ameliorated soon after splenectomy.

\section{DISCUSSION}

ANK1 mutations are responsible for the majority of all cases of HS. Patients carrying $A N K 1$ mutations mainly exhibit an autosomal dominant pattern of inheritance, but some of these mutations are inherited in an autosomal recessive pattern $[8,9]$. These mutations cover the entire ANK1 gene, including the promoter region, and de novo mutations occur with high frequency [10]. To date, a total of 59 mutations are included in the HGMD public database. Small deletion and nonsense mutations account for more than $50 \%$ of all mutations in the $A N K 1$ gene. Seven splicing mutations, occurring in IVS1, 16, 20, 22, 28 , and 38 , are included in the HGMD database. In this study, patients displayed an autosomal dominant pattern of inheritance. Both the girl and her affected father carried a heterozygous $A N K 1$ IVS3-2A $>C$ mutation identified by NGS and confirmed by Sanger sequencing.

NGS has been widely used for genetic diagnosis of hereditary red blood cell membrane disorders, including HS [11]. In this study, the mean depth was 2,282 folds, and $93.49 \%$ target bases were covered at least 500 times. Target bases covered fewer than 20 times and the uncovered regions were analyzed using Sanger sequencing. These results confirmed the presence of the variants identified by NGS.

The major form of Ankyrin includes an 89-kd $\mathrm{N}$-terminal region composed of 24 conserved repeats, 
a membrane domain containing binding site for band 3, a 62-kd central domain containing binding sites for spectrin and vimentin, and a 55-kd C-terminal regulatory domain [12]. In this family, a $A N K 1$ IVS3-2A $>$ C mutation occurred in the $\mathrm{N}$-terminus region. Sequencing of cDNA transcribed from mRNA isolated from patient-derived peripheral blood mononuclear cells revealed that the ANK1 IVS3-2A $>$ C mutation resulted in the skipping of exon 4 in the $A N K 1$ gene. Exon 4 deletion did not affect the reading frame of the $A N K 1$ gene, but it may produce a mutant protein that lacks the 33 amino acids encoded by exon 4 . The $A N K 1$ IVS3-2A $>$ C mutation may disrupt the binding of band 3 protein. In silico splicing prediction programs also revealed that the $A N K 1$ IVS3-2A $>\mathrm{C}$ mutation weakened the 3 ' splice-site consensus sequence of exon 4.

Splenectomy is therapeutic for patients with HS after the age of $6[13,14]$. After surgery, the anemia and jaundice of the 7-year-old girl were ameliorated within one week, although increased platelet counts due to the splenomegaly continued for two weeks. Her father, who received a splenectomy when he was young, is now asymptomatic.

In summary, our results demonstrate that an $A N K 1$ IVS3-2A $>$ C mutation may lead to exon 4 skipping of the $A N K 1$ gene and may be responsible for HS in two patients from a Chinese family.

\section{MATERIALS AND METHODS}

\section{Subjects}

Samples from a 7-year-old girl suspected of HS and her parents were collected. The girl received a splenectomy due to severe anemia. Her father also received this surgery when he was 10 years old. Osmotic fragility was increased, and G6PD activity was normal. Genetic mutation screens for $\alpha$ - and $\beta$-thalassemia was negative. Autoimmune antibody tests were negative. Spherical-shaped erythrocytes were found in the peripheral blood film. Written informed consent was obtained from all subjects, and this work was formally approved by the Ethics Committee of Tongji Hospital, Tongji Medical College, Huazhong University of Science and Technology. All procedures were performed in accordance with the approved guidelines.

\section{Ampliseq NGS panel design}

A custom NGS panel covering all exons and adjacent introns of the $A N K 1, S L C 4 A 1, S P T B, S P T A 1$, and EPB42 genes was designed using the Ampliseq tool (http://www.ampliseq.com) with coverage of $96.71 \%$, $96.53 \%, 99.54 \%, 100 \%$, and $100 \%$, respectively. The uncovered sequences were amplified and sequenced by Sanger sequencing.

\section{NGS}

DNA libraries were built according to the Ampliseq ${ }^{\mathrm{TM}}$ Library Preparation Kit 2.0 using $10 \mathrm{ng}$ of input DNA from PBMC in two primer-pools with 118 and 115 amplicons. The PCR products of the two pools were mixed together and purified using AMPure XP beads (Beckman Coulter, Brea, CA, USA) and quantified using the Ion Library TaqMan ${ }^{\mathrm{TM}}$ Quantitation Kit. Prepared libraries were pooled in equal amounts and diluted to a concentration of $100 \mathrm{pM}$. Samples $(2 \mu \mathrm{L})$ of the pooled library was further enriched using a One-Touch machine and ES Instrument using the Ion PGM ${ }^{\mathrm{TM}} \mathrm{Hi}-\mathrm{Q}^{\mathrm{TM}} \mathrm{OT} 2 \mathrm{Kit}$. Enriched products were sequenced using the Ion Torrent PGM Hi-Q Sequencing Kit on PGM using the Ion $316^{\mathrm{TM}}$ chip.

\section{Bioinformatic analysis}

Trimmed raw data were aligned to the hg19 human reference genome. Coverage and variant identification analysis was performed on the Ion Torrent Server 4.4.2. Variants were annotated with the Ion Reporter 5.0 software according to the nomenclature recommended by the Human Genome Variation Society (HGVS, http:// www.hgvs.org). All identified variants and sequences with less than $20 \times$ coverage depth were visually verified using the Integrative Genomics Viewer 2.3.8 (Broad Institute, Cambridge, MA, USA). Clinical significance of selected variants was made according to the $1000 \mathrm{G}$ (http://asia.ensembl.org/index.html), ExAC (http://exac. broadinstitute.org/), and HGMD (http://www.hgmd.cf.ac. uk/ac/index.php) databases.

\section{Sanger sequencing}

Sanger sequencing was performed as previously described. Briefly, genomic DNA was extracted from PBMCs using the QIAamp DNA blood mini kit. Coding exons and splice junctions of HS-related genes were amplified for selected mutations, failed amplified or uncovered regions. RNA was extracted from PBMCs using Trizol and reversely transcribed into cDNA for sequencing. Sanger sequencing was performed bidirectionally on an ABI 3500 Dx. NM_001142446.1 was used as a reference transcript for the $A \bar{N} K 1$ gene. Primers used to amplify the region spanning exon 2 to 6 of the ANK1 gene from cDNA are as follows: $A N K 1$ E2-6 RT F (381 bp), 5'-CAGAATGGGTTGAATGGCT-3'; ANK1 E2-6 RT R (381 bp), 5'-CTTGGTGCCGTAGTTGATG-3'. Primers used to amplify exon 4 from 
DNA are as follows: ANK1 E4 F (545 bp), 5'-GTCATAGAGAACAGGCTGGATC-3'; ANK1 E4 R (545 bp), 5'-GGCTTAGGTCCAAAACAATTC-3'.

\section{Splicing predictions}

To assess the presumptive effects of the splicing mutation, two in silico prediction tools were applied: the GENIE program (http://rulai.cshl.edu/new alt exon $\mathrm{db}$ /HTML/score.html) and MES using the Weight Matrix Model (http://genes.mit.edu/burgelab/maxent/ Xmaxentscan_scoreseq_acc.html) [15]. The high value indicates a high possibility of being a splicing site.

\section{ACKNOWLEDGMENTS AND FUNDING}

This work was supported by the National Natural Science Foundation of China (No. 81500925).

\section{CONFLICTS OF INTEREST}

There is no conflict of interest.

\section{REFERENCES}

1. Perrotta S, Gallagher PG, Mohandas N. Hereditary spherocytosis. Lancet. 2008; 372: 1411-26.

2. Wang C, Cui Y, Li Y, Liu X, Han J. A systematic review of hereditary spherocytosis reported in Chinese biomedical journals from 1978 to 2013 and estimation of the prevalence of the disease using a disease model. Intractable Rare Dis Res. 2015; 4: 76-81.

3. Manciu S, Matei E, Trandafir B. Hereditary Spherocytosis Diagnosis, Surgical Treatment and Outcomes. A Literature Review. Chirurgia (Bucur). 2017; 112: 110-6.

4. Narla J, Mohandas N. Red cell membrane disorders. Int J Lab Hematol. 2017; 39 Suppl 1: 47-52.

5. Miya K, Shimojima K, Sugawara M, Shimada S, Tsuri H, Harai-Tanaka T, Nakaoka S, Kanegane H, Miyawaki T, Yamamoto T. A de novo interstitial deletion of $8 \mathrm{p} 11.2$ including ANK1 identified in a patient with spherocytosis, psychomotor developmental delay, and distinctive facial features. Gene. 2012; 506: 146-9.

6. Mohler PJ, Bennett V. Defects in ankyrin-based cellular pathways in metazoan physiology. Front Biosci. 2005; 10: 2832-40.

7. Del Orbe Barreto R, Arrizabalaga B, De la Hoz AB, Garcia-
Orad A, Tejada MI, Garcia-Ruiz JC, Fidalgo T, Bento C, Manco L, Ribeiro ML. Detection of new pathogenic mutations in patients with congenital haemolytic anaemia using next-generation sequencing. Int J Lab Hematol. 2016; 38: 629-38.

8. Eber SW, Gonzalez JM, Lux ML, Scarpa AL, Tse WT, Dornwell M, Herbers J, Kugler W, Ozcan R, Pekrun A, Gallagher PG, Schroter W, Forget BG, et al. Ankyrin-1 mutations are a major cause of dominant and recessive hereditary spherocytosis. Nat Genet. 1996; 13: 214-8.

9. Nakanishi H, Kanzaki A, Yawata A, Yamada O, Yawata Y. Ankyrin gene mutations in japanese patients with hereditary spherocytosis. Int J Hematol. 2001; 73: 54-63.

10. Gallagher PG. Hematologically important mutations: ankyrin variants in hereditary spherocytosis. Blood Cells Mol Dis. 2005; 35: 345-7.

11. He Y, Jia S, Dewan RK, Liao N. Novel mutations in patients with hereditary red blood cell membrane disorders using next-generation sequencing. Gene. 2017; 627: 55662.

12. Peters LL, Lux SE. Ankyrins: structure and function in normal cells and hereditary spherocytes. Semin Hematol. 1993; 30: 85-118.

13. Jankulovski N, Antovic S, Kuzmanovska B, Mitevski A. Splenectomy for haematological disorders. Pril (Makedon Akad Nauk Umet Odd Med Nauki). 2014; 35: 181-7.

14. Bolton-Maggs PH, Stevens RF, Dodd NJ, Lamont G, Tittensor P, King MJ, General Haematology Task Force of the British Committee for Standards in Haematology. Guidelines for the diagnosis and management of hereditary spherocytosis. Br J Haematol. 2004; 126: 455-74.

15. Yeo G, Burge CB. Maximum entropy modeling of short sequence motifs with applications to RNA splicing signals. J Comput Biol. 2004; 11: 377-94. 